\title{
Video Watermarking by Using Geometric Warping Without Visible Artifacts
}

\author{
Dima Pröfrock, Mathias Schlauweg, and Erika Müller \\ Institute of Communications Engineering, \\ University of Rostock, \\ Rostock 18119, Germany, \\ \{dima.proefrock, mathias.schlauweg, erika.mueller\}@uni-rostock.de
}

\begin{abstract}
Our paper proposes an enhanced video watermarking approach. The fundamental idea is to use geometric warping for watermarks with high predictable robustness to lossy compression. We explain the basic watermarking approach which uses a block based statistic (Normed Centre of Gravity - NCG) to describe the geometric structure of blocks. The NCG also is used to choose robust blocks. To embed the watermark information the chosen blocks are changed by geometric warping. To extract the watermark, the original video is not necessary. The NCG is used to detect the watermarked blocks and compute the embedded watermark bit. In some cases, the independent geometric warping of blocks which contain the same object results in visible artifacts. We propose to link blocks in space and time to block groups. In contrast to the basic approach, the blocks of one block group can be warped in dependence on each other. Thus, the visible artifacts are prevented.
\end{abstract}

\section{Introduction}

Current information technologies are based more and more on digital multimedia data. The use of digital data instead of analogue data offers many advantages. A lot of digital data can be produced in a very short time and it becomes more and more trivial to edit and finish the data. As opposed to analogue data, digital data can be endlessly copied without any loss of quality. However, the technologies to manipulate and copy data are often used in an illegal manner.Hence, there is a growing importance of applications such as data authentication, copyright and data hiding. Digital watermarking offers contributions in these fields. It describes techniques to embed additional information, the watermark, into digital data [1].Transparency, robustness and capacity are some important and application dependent properties of watermarking. Especially in the case of video watermarking, watermarks with high robustness to lossy compression are required. Generally, videos are compressed with lower data rates as single images. Hence, image watermarking techniques can not be automatically used for video watermarking. 
There are watermarking approaches that solve the problem by embedding the watermark into the compressed domain during or after the encoding process (e.g., [2], [3]). This method has the advantage that the watermarking process is not influenced by the compression. However, the watermarks are generally not robust to a transcoding of the video. This paper presents a watermark approach in the uncompressed domain. The uncompressed video is watermarked and can be compressed with different compression algorithms and data rates. The watermark is robust to the compression.

Generally, the watermark is embedded into the irrelevant information of video data to be invisible. This results in problems because compression algorithms try to remove irrelevant information. In [4], we propose a basic watermarking approach which embeds the watermark in the relevant information of videos but in an imperceptible manner. This approach is based on geometric warping of blocks. Because common compression algorithms are PSNR (Peak Signal to Noise Ratio)-optimized, they try to maintain the geometric structure. With this approach a high robustness to the new H.264/AVC compression standard can be achieved. At present, the new H.264/AVC standard, developed for a broad range of applications, provides the highest coding performance [5], [6]. Because this, especially a H.264/AVC compression is suitable to verify the robustness of this new watermarking approach. However, in some cases the basic watermarking process described in [4] results in visible artifacts. We propose a method to prevent these artifacts and improve the video quality.

In this paper, we present an improvement of a basic watermarking approach. Firstly, the fundamental idea of watermarking by geometric warping is described. Afterwards, the principle of the basic approach is explained. Therefore, a statistic to describe the object borders in blocks is introduced. We propose a method to choose robust blocks for watermarking and to detect watermarked blocks even after lossy compression. The enhanced embedding process is explained. The next section describes the way to reduce the artifacts of the basic approach. The several subsections contain information about the reasons for the artifacts, the approach to prevent them, an algorithm to realize the approach as well as results and analyzes of the basic approach enhancement. 


\section{Watermarking by Geometric Warping}

Commonly, the watermark is embedded in the perceptual invisible part of the video. The compression algorithms try to remove the perceptual invisible part of the video. Generally, both systems use the PSNR to measure the perceptual quality degradation. Because this, both systems use the same definition for relevant video data. This implies a contradiction. We can not embed a watermark in video parts which are removed during the compression process. We can solve this problem as shown in Figure 1.

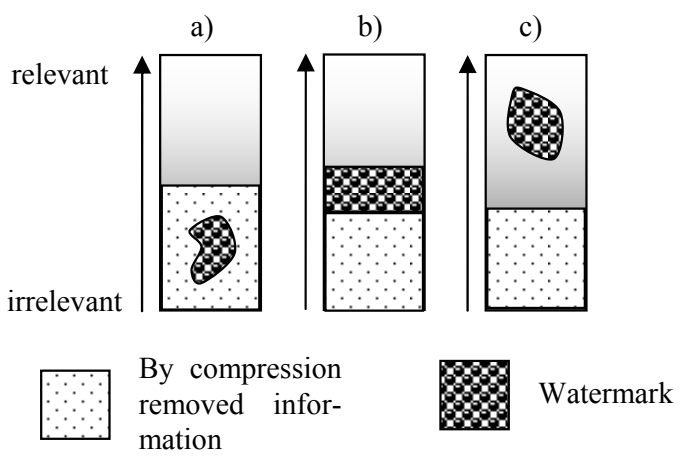

Fig. 1. Watermark embedding by using gaps of the compression algorithm a), defined embedding strength b) or by using the relevant video information c).

The optimal way to embed the watermark with robustness to lossy compression is to embed the watermark in the relevant part of video data. Because common compression algorithms are PSNR-optimized, the relevance is defined by the PSNR. We propose in [4] to change the geometric structure of the video to embed the watermark. Because the PSNR-optimization, compression algorithms try to maintain the geometric structure. At the same time, the geometric embedding process can be imperceptible. For example see Figure 2. The PSNR between both images is $27.7 \mathrm{~dB}$. However, the difference is imperceptible.

a)

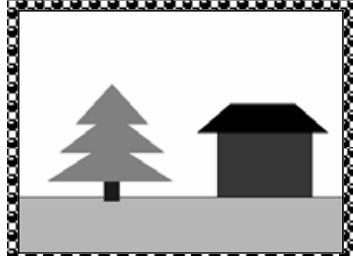

b)

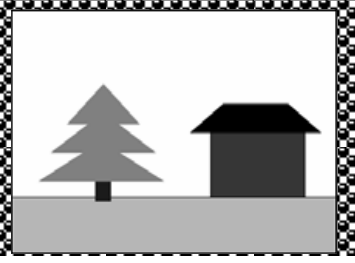

c)

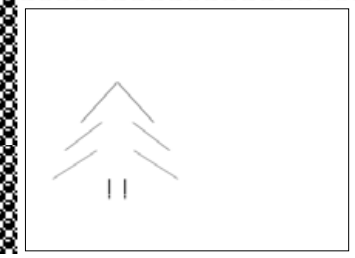

Fig. 2. Original image a), by geometric warping changed image b) and difference image c). The geometric warping process moved the tree by some pixel to the left side. 


\section{The Basic Approach}

\subsection{The Normed Centre of Gravity (NCG)}

To realize the geometric warping process, we introduce in [4] a new statistic, the Normed Centre of Gravity (NCG). The NCG is similar to the gravity centre of one block. However, it is independent from the block borders and every grayvalue of the pixel has the same influence to the NCG. The NCG is computed in the following way.
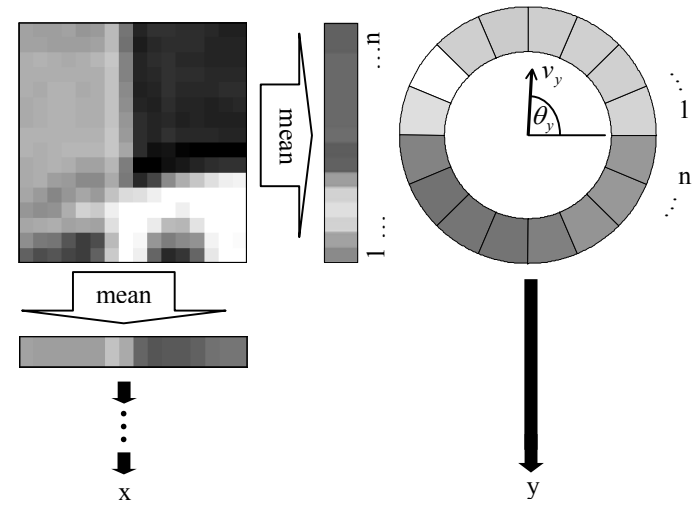

Fig. 3. Computing scheme for the NCG x,y-coordinates.

First, the mean values of the rows and columns of the block are computed. The results are two vectors $\underline{m}_{x}$ and $\underline{m}_{y}$. The vector $\underline{m}_{x}$ is used to compute the x-coordinate of the NCG, the vector $\underline{m}_{y}$ is used to compute the y-coordinate. Therefore, the two vectors of mean values are arranged in two circles. Now, the two-dimensional vector $\underline{v}_{k}(k=\mathrm{x}$ or $\mathrm{y})$ is computed.

$$
\underline{v}_{k}=\left(\begin{array}{c}
\sum_{i=1}^{n} \underline{m}_{k}(i) \cdot \cos \left(\frac{\pi}{n}+\left((i-1) \cdot\left(\frac{2 \cdot \pi}{n}\right)\right)\right) \\
\sum_{i=1}^{n} \underline{m}_{k}(i) \cdot \sin \left(\frac{\pi}{n}+\left((i-1) \cdot\left(\frac{2 \cdot \pi}{n}\right)\right)\right)
\end{array}\right)
$$

For each vector, the vector angles $\Theta_{x}, \Theta_{y}$ and the vector lengths $L_{x}, L_{y}$ are computed. The vector angles are used to compute the $\mathrm{x}, \mathrm{y}$-coordinates of the NCG.

$$
x=\frac{n \cdot \Theta_{x}}{2 \cdot \pi} \quad y=\frac{n \cdot \Theta_{y}}{2 \cdot \pi}
$$




\subsection{Choosing and Detecting Robust Blocks}

To embed a watermark bit, robust blocks are chosen. Robust blocks are blocks with a high $L=\sqrt{L_{x}^{2}+L_{y}^{2}}$. The spatial position of object borders inside these blocks is very robust to lossy compression. To choose robust blocks a threshold is used (see [4]). For example, blocks with $L>430$ are robust to H.264/AVC compression with very low data rates. However, $L$ is slightly influenced by compression. Hence, it is a problem to find the correct blocks for watermark extraction after compression. To solve this problem we create a gap as shown in figure 4 b). In this example, blocks with $330<L_{\text {original }}<=430$ are changed so that $L_{\text {new }}=330$ and blocks with $430<L_{\text {original }}<530$ are changed so that $L_{n e w}=530$. This gap is sufficient for a correct detection of blocks even after compression with low data rates.
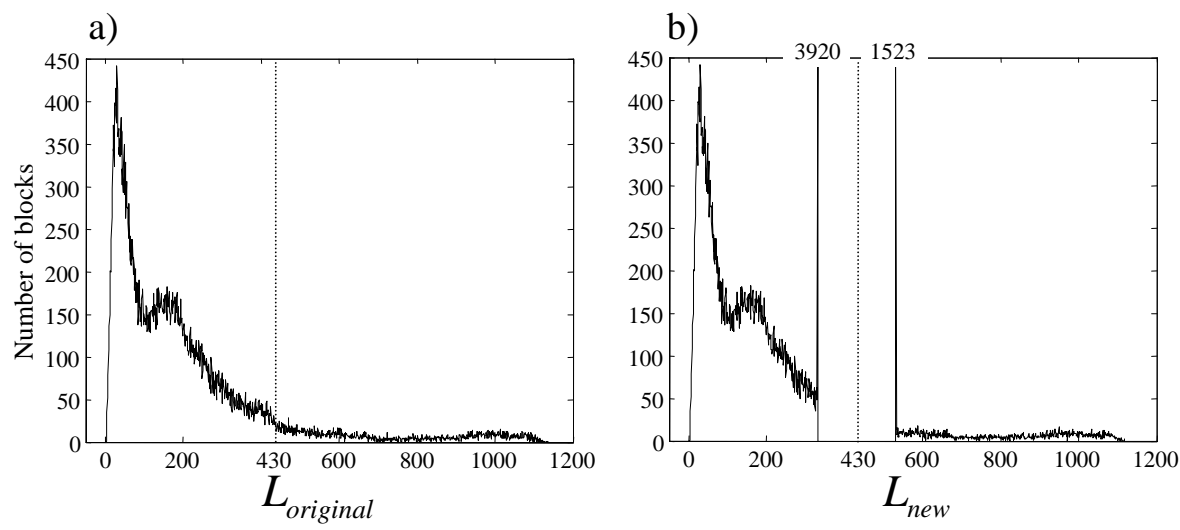

Fig. 4. Creating a gap in Video "Bus" for correct detection of robust and non-robust blocks around the threshold 430 with a) original distribution of $L$ and b) distribution after creating a gap.

\subsection{Enhanced Embedding Process}

In [4], we use a QIM approach [7] and quantize the NCG x,y-coordinates of robust blocks. Therefore we can distinguish 3 cases. There are blocks with robust x-coordinates (high $L_{x}$ ), robust y-coordinates (high $L_{y}$ ) and blocks with robust x,y-coorinates (high $L_{x}$ and $L_{y}$ ). We use a hard decision to embed the watermark bit. In dependence on these cases we quantize the $\mathrm{x}-, \mathrm{y}$ - or $\mathrm{y}, \mathrm{x}$-coordinates. To extract the bit we have to know which coordinate was used for embedding. However, after lossy compression $L_{x}$ and $L_{y}$ are slightly changed. Hence, the decision for the quantized coordinate can fail at the extraction process. 
To solve this problem we don't use the NCG x,y-coordinate directly to embed the bit. The $\mathrm{x}, \mathrm{y}$-coordinates are mapped to an adaptive quantization lattice that is defined by $L_{x}, L_{y}$ and the variable quant. quant is inversely proportional to the quantization step size and defines the embedding strength. Mapping the $\mathrm{x}, \mathrm{y}$-coordinates to the adaptive quantization lattice is described by equation 6 and yields the value s. The different robustness of the NCG x- and y-coordinate is considered in equation 4 . The influence of lossy compression to $L_{x}$ and $L_{y}$ is reduced by using equation 3. Equation 5 enables a linear mapping of the $\mathrm{x}, \mathrm{y}$-coordinates which is independent of block borders.

$$
\begin{aligned}
& f_{f}(a, b)=\frac{\left(e^{\frac{1.8 \cdot a}{1200}}\right)}{1+\left(1-\left|\frac{a-b}{1200}\right|\right) \cdot\left(e^{\frac{1.8 \cdot a}{1200}}-e^{1.8}\right)} \\
& f_{x}\left(L_{x}, L_{y}\right), f_{y}\left(L_{x}, L_{y}\right)=\left\{\begin{aligned}
L_{x} \leq L_{y} \rightarrow \begin{array}{l}
f_{x}=f_{f}\left(L_{x}, L_{y}\right) \cdot \frac{L_{x}}{L_{x}+L_{y}} \\
f_{y}=1-f_{x}
\end{array} \\
L_{x}>L_{y} \rightarrow \begin{array}{l}
f_{y}=f_{f}\left(L_{y}, L_{x}\right) \cdot \frac{L_{y}}{L_{x}+L_{y}} \\
f_{x}=1-f_{y}
\end{array}
\end{aligned}\right. \\
& \operatorname{tria}(a, q)=\left\{\begin{array}{l}
\operatorname{modulo}\left(a, \frac{16}{q}\right) \leq \frac{8}{q} \rightarrow \operatorname{tria}=\frac{\operatorname{modulo}\left(a, \frac{16}{q}\right) \cdot q}{16} \\
\operatorname{modulo}\left(a, \frac{16}{q}\right)>\frac{8}{q} \rightarrow \operatorname{tria}=\frac{16-\operatorname{modulo}\left(a, \frac{16}{q}\right) \cdot q}{16}
\end{array}\right. \\
& s(x, y)=f_{x}\left(L_{x}, L_{y}\right) \cdot \operatorname{tria}(x, \text { quant })+f_{y}\left(L_{x}, L_{y}\right) \cdot \operatorname{tria}(y, \text { quant })
\end{aligned}
$$

The used quantization lattice is self-adapting on each block (see Figure 5 II). The quantization lattice consists of periodically arranged minima $(s=0)$ and maxima $(s=1)$. To embed a watermark bit we don't quantize the NCG $\mathrm{x}, \mathrm{y}$-coordinates but we move the $\mathrm{x}, \mathrm{y}$-coordinates on the quantization lattice to the next minimum (to embed a watermark bit ' 0 ') or maximum (to embed a watermark bit '1'). For example see Figure 5. In column II the quantization lattice of blocks of column I with the original NCG x,y-coordinates (marked with a cross) can be seen. The blocks of column I are changed by geometric warping to embed a watermark bit ' 0 ' (column III). The result can be seen in Figure 5 column IV. The NCG x,y-coordinates are at the minima (black areas represent the minima, white areas the maxima ) on the quantization lattice. To extract the embedded bit, the original block is not required. Only the NCG x,y-coordinates have to be mapped to the quantization lattice of the changed block. The use of equation 6 delivers a value between 0 and 1 . The watermark bit ' 0 ' is extracted if $0 \leq s<0.5$, the watermark bit '1' is extracted if $0.5 \leq s \leq 1$. 


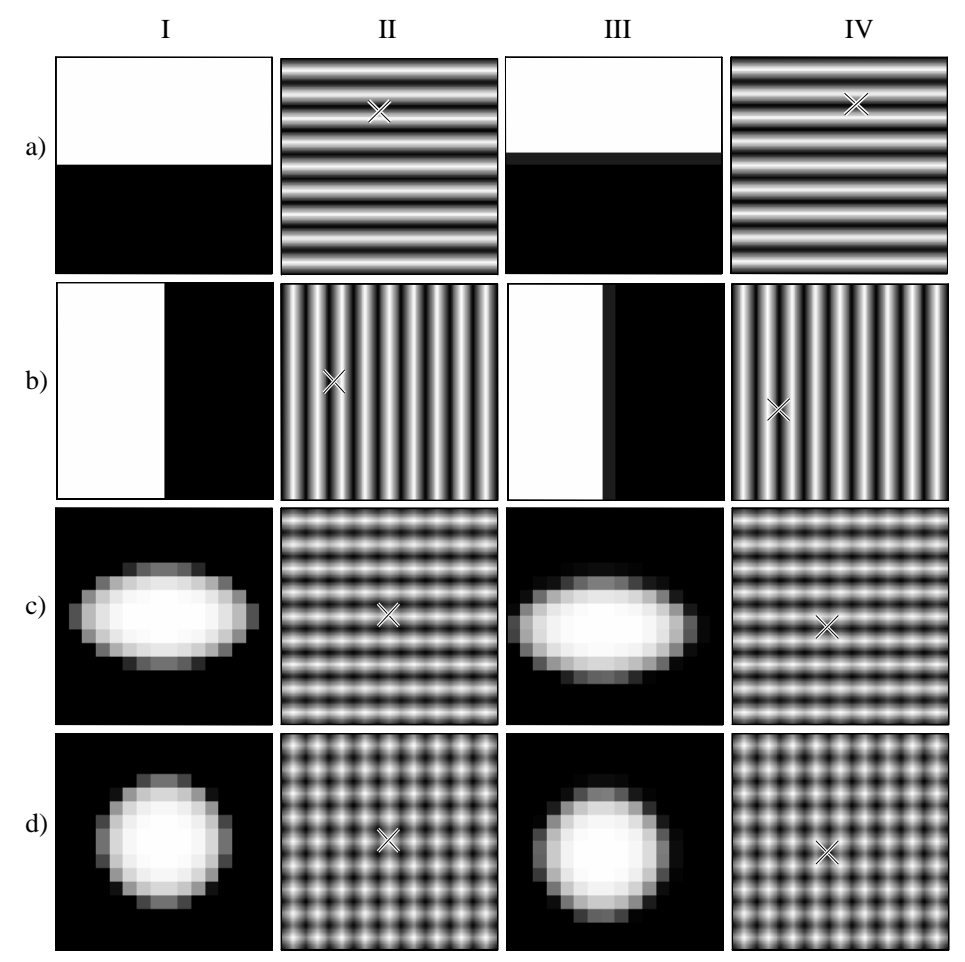

Fig. 5. Enhanced embedding process with a) robust $y$-coordinate, b) robust $x$ coordinate, c) robust $\mathrm{y}$-, slightly robust $\mathrm{x}$-coordinate and $\mathrm{d}$ ) robust $\mathrm{x}, \mathrm{y}$-coordinate. Column I shows the original blocks, column II shows the quantization lattice with NCG x,y-coordinates, column III shows watermarked blocks and column IV shows the new quantization lattice of watermarked blocks with new NCG x,y-coordinates.

\subsection{Achievable Robustness}

Because the PSNR-optimization, we assume that the position of object borders is very robust to lossy compression. The position of object borders is described by the NCG and mapped to an adaptive quantization lattice yielding the value s. The strength of the object border is described by the $L$. Higher $L$ yields a stronger object border. Figure 6 shows the robustness of the embedded watermark information bits to H.264/AVC compression. Different $L$ and a block size of $16 \times 16$ pixels are used. The embedding process changes the position of the object borders by maximal 0.5 pixels. 
a)

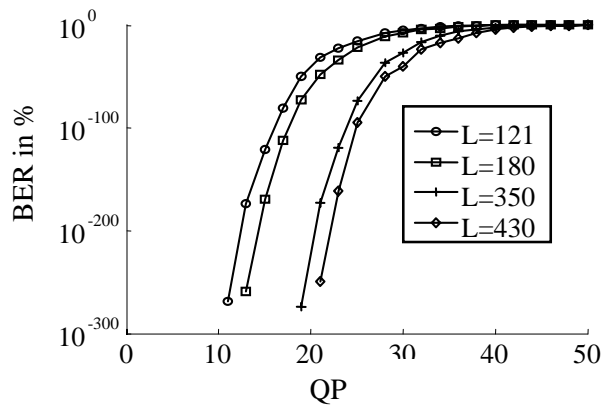

b)

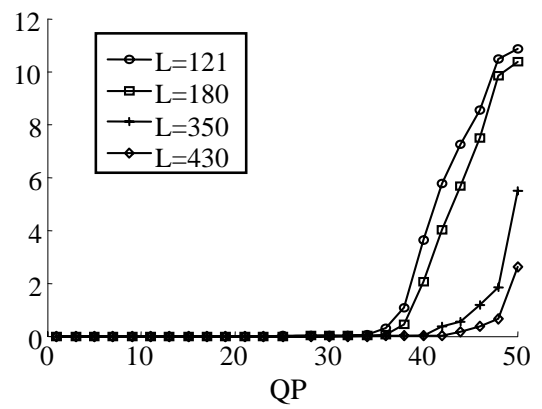

Fig. 6. Bit error rate (BER) of geometric warping based watermarking with logarithmic and linear BER axes. $Q P$ represents the H.264/AVC quantization parameter. A higher $Q P$ yields a lower compression data rate.

The relationship between the H.264/AVC quantization parameter $Q P$ and the resulting PSNR of the compressed video is shown in figure 7.

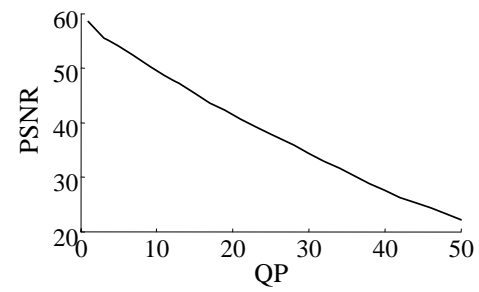

Fig. 7. Reduced quality of the reference video "Bus" caused by lossy H.264/AVC compression.

As shown in figure 6, the robustness of geometric warping based watermarking to lossy compression is very high even on a strong lossy compression quality distortion. At the same time, the capacity is relatively high (see figures 8 and $16)$.

\subsection{Results of the Basic Approach}

The approach is tested by embedding watermarks in standard videos "Bus", "Horse", "Horse2", "Waterfall" and "Foreman". The video resolution is 352x288 pixels. The embedding strength is quant $=16$ with a block size of $16 \times 16$. This is equivalent to a quantization of NCG x,y-coordinates with a quantization step size of one. The watermark is embedded with different robustness to H.264/AVC compression. A higher $Q P$ yields lower data rates and reduces the watermark capacity if we use the same embedding strength to embed the watermark. The 
capacity depends on the required robustness of the watermark and the video content. The watermark is embedded with robustness to H.264/AVC compression with $Q P_{\max }$ between 26 and 40. Figure 8 shows the results. As expected, the capacity of videos without distinct objects, such as "Waterfall", is lower than in videos with distinct objects, such as "Bus".

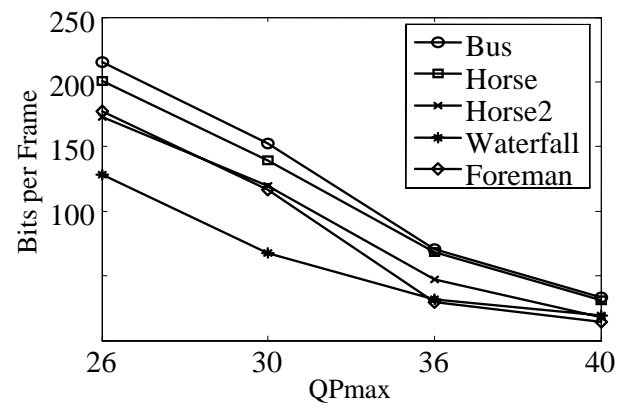

Fig. 8. Capacity in Bits per Frame. The embedding strength is quant $=16$. The watermarks are embedded with robustness to different H.264/AVC compression. $Q P_{\max }$ defines the highest $Q P$ at which the watermark can be extracted.

The watermark results in visible artifacts as shown in Figure 9 b) and c). However, the watermark is imperceptible if viewers don't compare pixels but see the video as a whole. The frame in Figure 9 a) contains 22 watermarked blocks. For example, the wooden bole in the bottom right corner contains six of them. But, without comparing the original pixels with the changed pixels nobody is able to notice these blocks.

a)

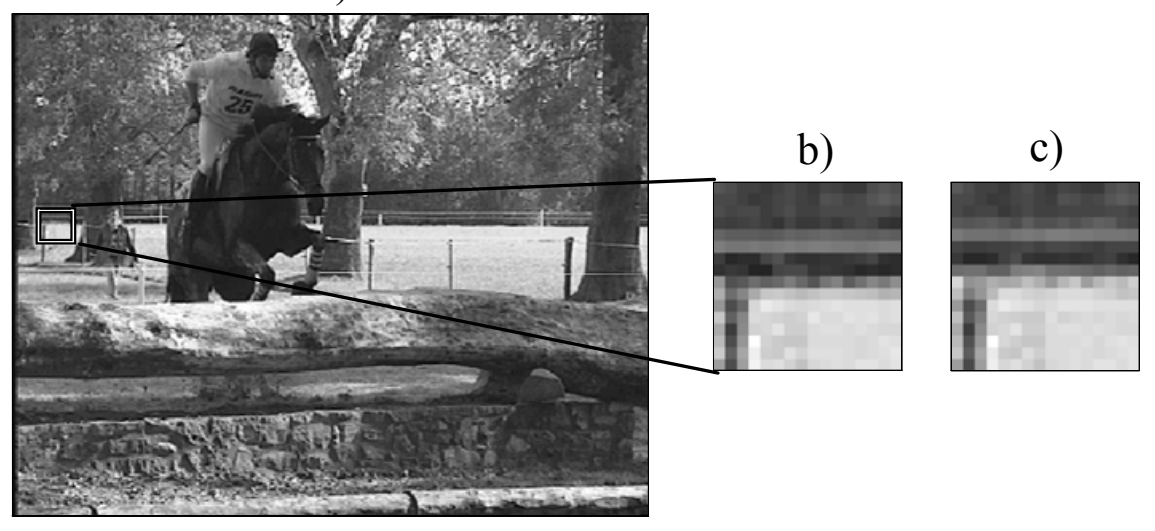

Fig. 9. Watermarked frame of "Horse" a), original block b) and watermarked block c). 


\section{Block-Linking for Improved Quality}

\subsection{Artifacts of the Basic Approach}

The embedded watermark is imperceptible and robust to lossy compression with low data rates. However, in some cases visible artifacts can be found after the watermarking process. There are two kinds of artifacts. If one edge passes several robust blocks which are changed by geometric warping to embed the watermark, visible steps on the edge are produced. Hence, steps on long edges in single frames are the first type of artifacts (see Figure 10). The second type of artifacts can appear, if several blocks in succeeding frames with the same block position are changed by geometric warping. Even if there are no visible differences between the original and the watermarked single frames, there are visible flicker-effects if the video is played.

a)

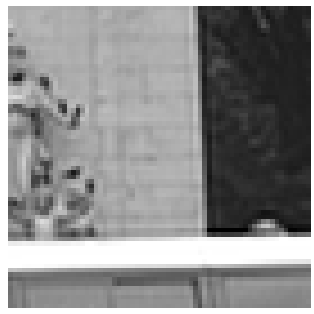

b)

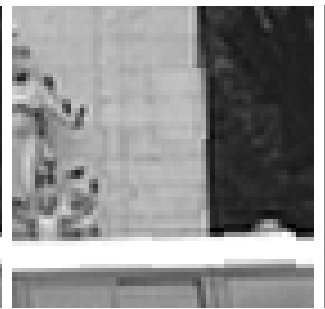

c)

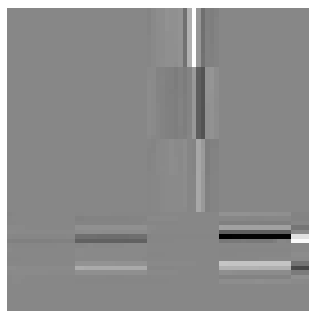

Fig. 10. Part of "Bus" a) original, b) watermarked with visible artifacts and c) difference image.

\subsection{Prevent Artifacts by Block Linking}

The artifacts are the result of the independent warping of the blocks. To solve this problem, we propose to link blocks which contain the same object to block groups. Now, the warping process of one block in one block group can consider the warping process of the other blocks of the same group. In this way, the artifacts can be prevented.

Linking Process To prevent artifacts and flicker-effects, we have to consider the video as a 3D-space. Two dimensions in space (coordinates $\mathrm{x}, \mathrm{y}$ ) and one dimension in time (frame number, coordinate t). The linking process uses neighboring robust blocks. It is probably that neighboring blocks contain the same 
object. Hence, robust blocks which are neighbors in spatial as well as in temporal position are linked to block groups. Figure 11 shows some block groups of the first 10 frames of the video "Bus". It can happen that one block group contains more than one object. However, this is not a disadvantage for preventing artifacts.

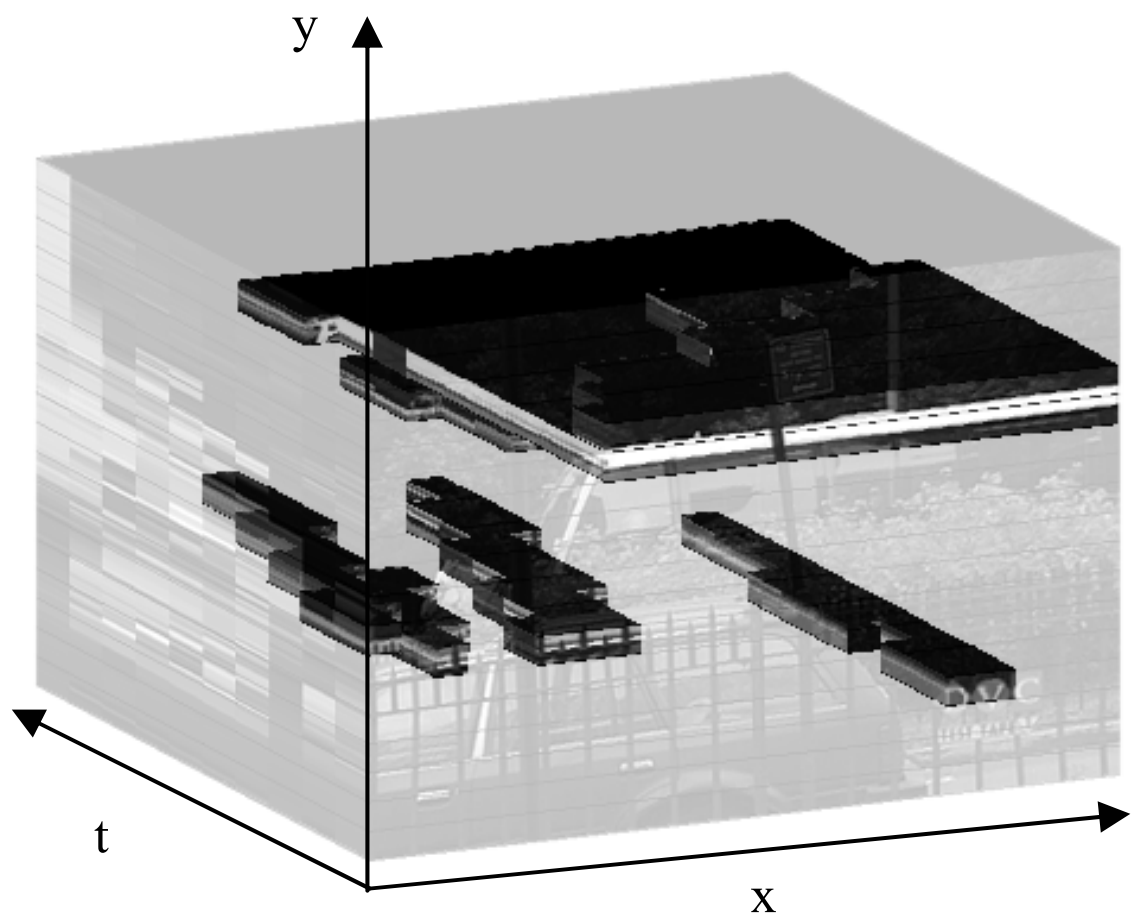

Fig. 11. Some block groups of the first 10 frames of "Bus".

Principle of Preventing Artifacts To prevent the artifacts, we do not embed a watermark bit in every block of one block group. We define a minimum spatial and temporal distance $d$ between blocks which will be watermarked. The blocks of one block group which are not chosen for watermarking are used to create a smooth transition between the watermarked blocks. For example see Figure 12. Figure 12 shows one black and one white object which are divided into 7 blocks. Embedding a watermark by geometric warping in each block inducts visible artifacts (Figure $12 \mathrm{~b}$ ). The artifacts are reduced by choosing only the block 1, 4 and 7 for watermarking (Figure $8 \mathrm{c}$ ) and using the blocks 2, 3, 5 and 6 to create a smooth transition between the watermarked blocks (Figure $12 \mathrm{~d}$ ). 
a)

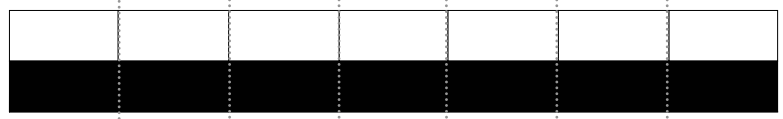

b)

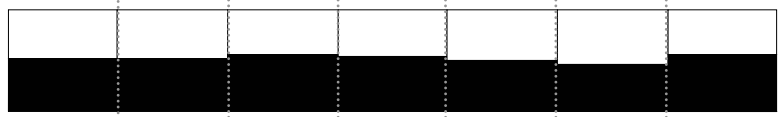

c)

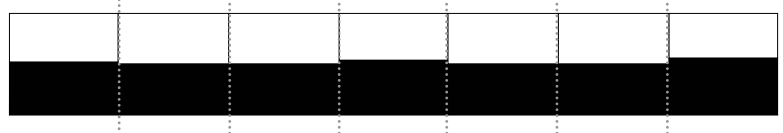

d)

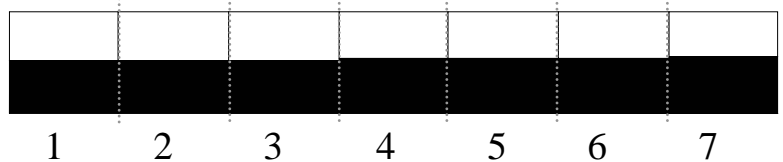

Fig. 12. Reducing artifacts by using a smooth transition between watermarked blocks. Therefore is exhibited a) blocks without watermark, b) watermark in every block, c) watermark in block 1, 4, 7 and d) watermark in block 1, 4, 7 by using block 2, 3, 5, 6 for smooth transitions.

Algorithm for Choosing Blocks Only some blocks of one block group have to be chosen for watermarking to realize the principle outlined in 3.3. To enable the watermark detection, this process has to be unambiguous. To prevent the artifacts, the chosen blocks should maintain a minimum distance $d$ to each other. Investigation has shown that small-sized block groups can use a small minimum distance to prevent artifacts. However, bigger-sized block groups have to use a higher minimum distance to prevent artifacts. Hence, we adapt $d$ in each block group for each dimension whereas $d_{x}, d_{y}$ and $d_{t}$ represent the minimum distance for each dimension and $x_{\text {size }}, y_{\text {size }}$ and $t_{\text {size }}$ represent the block group size for each dimension. Investigations have shown that distance $d>0.5$ is suitable to prevent the artifacts.

$$
d_{i}=\left\lfloor\log \left(d \cdot\left(i_{\text {size }}-1\right)+1\right)+0.5\right\rfloor \quad(i=x, y \text { or } t)
$$

To achieve a maximal watermark capacity, as much as possible blocks of the block group have to be chosen for watermarking. Hence, the following algorithm has to find a maximum number of blocks in a block group which have a minimum distance to each other. We realize it in the following way:

1. The block group is chosen. Block groups have arbitrary forms which depend on video content. For example see Figure 13 a.

2. For each dimension $\mathrm{x}, \mathrm{y}$ and $\mathrm{t}$ of the block group a minimum distance $d_{x}$, $d_{y}$ and $d_{t}$ is computed (equation 7 ).

3. For each block, the numbers of blocks inside an ellipsoid are counted (Figure $13 \mathrm{a}, \mathrm{b})$. The semi-axes of the ellipsoids are defined by $d_{x}, d_{y}$ and $d_{t}$. The center of the current ellipsoid is defined by the current block. For better illustration Figure 13 shows the principle only for a $2 \mathrm{D}$ block group. 
4. The blocks with the least neighbors are chosen for watermarking (Figure 13 c). Are more than one chosen blocks inside an ellipsoid, only one of them (the first) is used for watermarking (Figure $13 \mathrm{~d}, \mathrm{e}$ ). This process is unambiguous.

5. The chosen blocks and all blocks within the ellipsoids around the chosen blocks will not be considered in the next steps.

6. The steps 3-4 are repeated until all blocks are chosen for watermarking or have a distance lower than the minimum to a chosen block (Figure $13 \mathrm{~d}-\mathrm{f}$ ).

a)
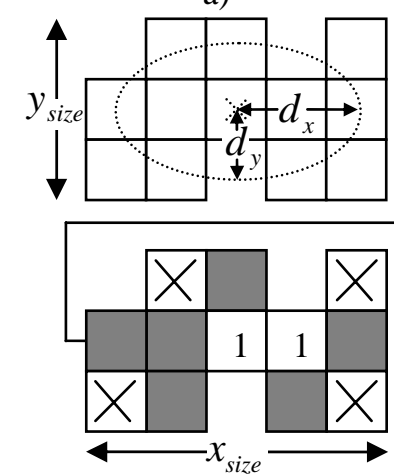

d) b)
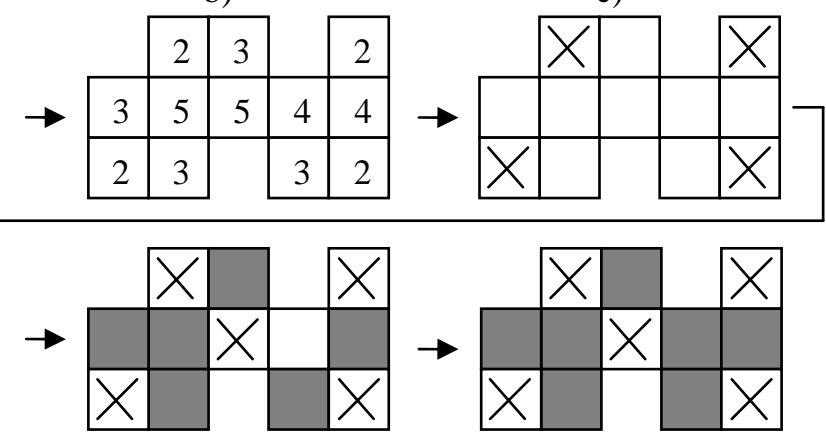

e)

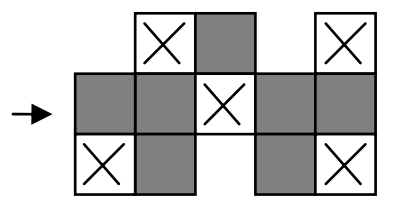

f)

Fig. 13. Example for choosing blocks for watermarking with a minimum distance in one block group with a) block group and ellipsoid, b) counted neighbors, c) first chosen blocks, d) counted remaining blocks, e) chosen blocks of the second iteration and f) all chosen blocks

Smooth Transition between Watermarked Blocks The chosen blocks are used for watermarking. The watermarking bits ' 0 '/'1' are embedded by moving the NCG $\mathrm{x}, \mathrm{y}$-coordinates on the quantization lattice to minima/maxima. This is done by geometric warping. The warping strength differs from block to block. Only the chosen blocks have to be warped. However, to reduce the visible effect of the geometric warping process, the other blocks of the block group also will be warped (Figure 14). The warping strength of these blocks depends on the warping strength of the watermarked blocks. It is computed by using a simple Gaussian filter. Thus, a smooth transition between watermarked blocks is achieved. 


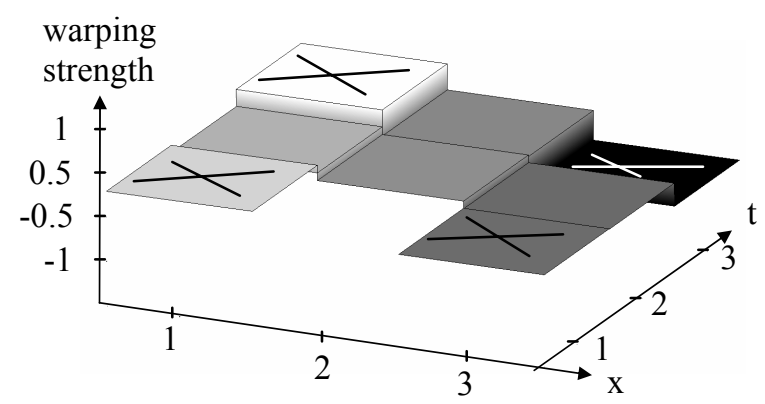

Fig. 14. A simplified example of warping strength in one block group. The watermarked blocks are labeled with a cross.

\subsection{Results of the Linking Process}

Linking blocks to block groups, choosing blocks with a minimum distance, watermarking them and using the other blocks for a smooth transition prevents the described artifacts. It can be clearly seen by comparing Figure 10 with Figure 15. The visible artifacts in single frames as well as the flicker-effects in succeeding frames are prevented.

a)

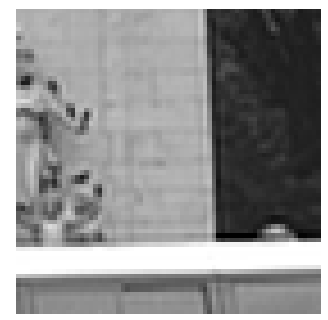

b)

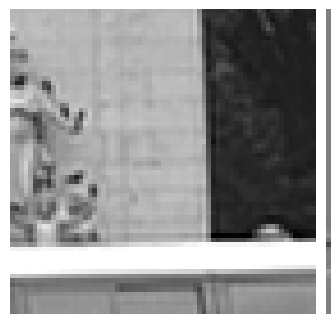

c)

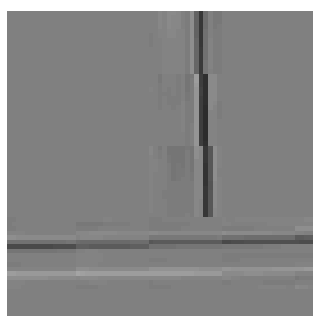

Fig. 15. Part of "Bus" a) original, b) watermarked without visible artifacts and c) difference image.

There is one disadvantage of this method. Without block linking, all robust blocks are used for watermarking. With block linking, only some blocks of a block group are used for watermarking. This results in a lower watermark capacity. Hence, with the block linking method and a minimum distance $d>0$ we can not embed the same number of bits as without block linking. The capacity depends not only on the minimum distance $d$ and the number of robust blocks. It also depends on the size and form of the block groups which depends 
on the video content. It can be seen clearly in Figure 8 and Figure 16. To test our approach, we embed watermarks in standard test videos "Bus", "Horse", "Horse2", "Waterfall" and "Foreman". The test conditions are the same as in section 3.5. Figure 8 shows the capacity by using all robust blocks. Figure 16 shows the capacity by using block linking and a minimum distance of 0.5 .

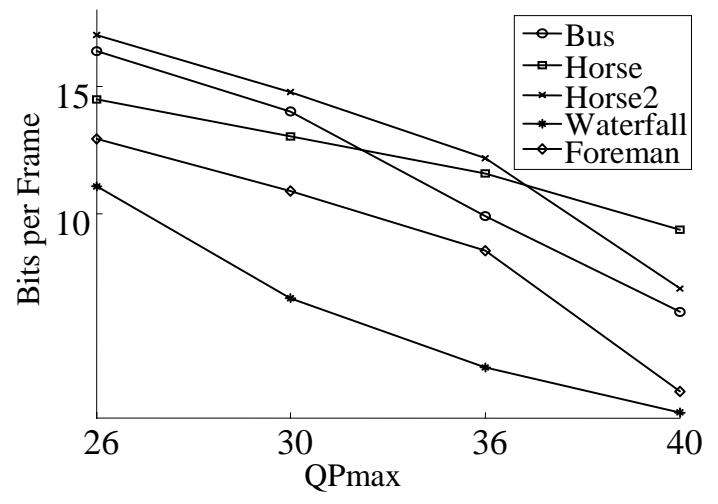

Fig. 16. Capacity in Bits per frame with block linking and a minimum distance of 0.5 .

Though "Bus" contains more robust blocks than "Horse2", the capacity with block linking is lower because "Bus" contains bigger block groups than "Horse2". As we said above (see 4.2), it is possible that one block group contain more than one object. However, several small block groups can contain more watermark bits (without visible artifacts) as one big block group. Hence, the watermark capacity can be increased in future works by changing the block linking method.

\section{Conclusions}

This paper presents an enhanced watermarking approach. The fundamental idea of using geometric warping for watermarks is explained. The basic watermarking approach is introduced. It uses a block based statistic (NCG) to describe the geometric structure of blocks. The NCG is also used to choose only robust blocks for watermarking. To detect robust blocks even after lossy compression we propose to change some blocks in a preprocessing step. The watermark information bits are embedded by moving the NCG x,y-coordinates on a block dependent quantization lattice. This is done by geometric warping of the block. The watermark is robust to lossy compression. The watermark capacity is analyzed. In some cases, the independent geometric warping of blocks which contain the same object results in visible artifacts. Especially on long edges, visible steps can appear. Additionally, flicker-effects in succeeding frames can be observed. 
In this paper we propose to link blocks in space and time to block groups. As opposed to the basic approach, the blocks of one block group can be warped in dependence on each other. Thus, the visible artifacts are prevented by our enhancement. However, the block linking approach reduces the watermark capacity. The capacity is analyzed and compared with the capacity of the basic approach.

\section{References}

1. Cox, J., Miller, M.: The First 50 Years of Electronic Watermarking. EURASIP J. of Applied Signal Processing 2002 (2002) 162-132

2. Qui, G., Marziliano, P., Ho A. T. S., He D., Sun Q.: A hybrid watermarking scheme for H.264/AVC video. Proc. of the ICPR 4 (2004) 865-868

3. Pröfrock, D., Richter, H., Schlauweg M., Müller E.: H.264/AVC video authentication using skipped macroblocks for an erasable watermark. Proc. of Visual Communications and Image Processing - VCIP 5960 (2005)

4. Pröfrock, H., Schlauweg M., Müller E.: A New Uncompressed-Domain Video Watermarking Approach Robust to H.264/AVC Compression. Proc. of Signal Processing, Pattern Recognition and Applications - SPPRA (2006)

5. Wiegand T., Sullivan G. J., Bjontegaard G., Luthra A.: Overview of the H.264/AVC Video Coding Standard. IEEE Transactions 13 (2003) 560-576

6. Draft ITU-T recommendation and final draft international standard of joint video specification (ITU-T Rec. H.264/ISO/IEC 14486-10 AVC, in Joint Video Team (JVT) of ISO/IEC MPEG and ITU-T VCEG, JVT-G050, (2003)

7. Chen B., Wornell G. W.: Quantization index modulation methods for digital watermarking and information embedding of multimedia. Journal of VLSI Signal Processing 27 (2003) 7-33 